\title{
Elucidation of structural, morphological, optical and photoluminescence properties of single and (In, Ga) co-doped ZnO nanocrystalline thin films
}

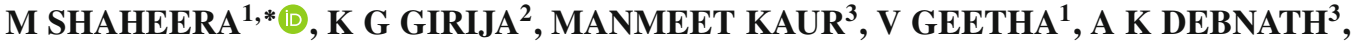 \\ R K VATSA ${ }^{2}$, K P MUTHE ${ }^{3}$ and S C GADKARI ${ }^{3}$ \\ ${ }^{1}$ Research Department of Physics, Government Victoria College, Palakkad 678001, India \\ ${ }^{2}$ Chemistry Division, Bhabha Atomic Research Centre, Mumbai 400094, India \\ ${ }^{3}$ Technical Physics Division, Bhabha Atomic Research Centre, Mumbai 400094, India \\ *Author for correspondence (kcmshahi@gmail.com)
}

MS received 29 October 2018; accepted 27 February 2019

\begin{abstract}
Single and co-doped $\mathrm{ZnO}$ thin films are currently under intense investigation and development for optoelectronic applications. Here in this study, pristine, indium-doped (IZO), gallium-doped (GZO) and co-doped (IGZO) ZnO thin films were deposited on a glass substrate using radio frequency magnetron sputtering. A comparative study of all the films was carried out on the basis of their various properties. The effect of single and co-doping on the structural (X-ray diffraction (XRD) studies and Raman studies), morphological (field emission scanning electron microscopy and energy dispersive X-ray spectroscopy studies) and optical properties (ultraviolet-visible (UV-Vis) and photoluminescence (PL)) of the deposited films was investigated. X-ray photoelectron spectroscopy (XPS) characterization was employed to analyse the surface chemical composition and bonding of the deposited film. From the XRD patterns, it was found that the films were highly crystalline in nature and preferentially oriented along the (002) direction with a hexagonal wurtzite structure, consistent with Raman analysis. IGZO films displayed a dramatic improvement in the surface morphology as compared with the single dopant films due to the compensation effect of gallium and indium doping which reduced the lattice strain. The XPS analysis confirmed the presence of the oxidized dopants in each film. All thin films have shown excellent optical properties with more than $90 \%$ transmission in the visible range of light. The blue-shift of the absorption edge accompanied by the increase of the optical band gap confirmed the Burstein-Moss effect. The UV PL peak originated from the near band edge emission of crystalline $\mathrm{ZnO}$, while the visible PL was associated with the radiative transition related to oxygen interstitial (Oi) defects in the $\mathrm{ZnO}$ structure.
\end{abstract}

Keywords. ZnO; co-doping; RF sputtering; XPS; photoluminescence.

\section{Introduction}

Zinc oxide $(\mathrm{ZnO})$ is a direct band gap $(\sim 3.37 \mathrm{eV}$ at room temperature) material and has a large exciton binding energy (B.E.) of $60 \mathrm{meV}$; hence it is widely used as an optoelectronic material $[1,2]$. Many other properties such as biocompatibility, thermal stability and relatively simple and low-cost production make it useful in a wide range of applications such as transparent conductive oxide for solar cells, threedimensional memory elements, thin film transistors, sensors, photo-detectors and light emitting diodes (LEDs) [3,4]. Doping of $\mathrm{ZnO}$ with suitable dopants is a well-known method for achieving the desired material properties. The idea of co-doping is not new. Co-doping allows the benefits of two dopants to be exploited simultaneously. The strengths of one dopant can compensate for the weaknesses of another. Many dopants have been used to improve the physical properties of $\mathrm{ZnO}$. The most common of these are the group 13 elements such as aluminium [5], gallium [6], indium [7], etc.
Some related studies based on $\mathrm{Al}$ and $\mathrm{Ga}$ co-doped $\mathrm{ZnO}$ nanostructures via a sol-gel route to modify their electrical and sensing properties have been carried out by Hayder et al [8]. While Benhaliliba et al [9] synthesized $\mathrm{ZnO}$ thin films which were doped separately with $\mathrm{Al}$ and In and these films exhibited good electrical conductivity and high optical transmittance in the visible region. These trivalent elements by replacing the $\mathrm{Zn}$ atom in the parent crystal structure modify the $\mathrm{ZnO}$ lattice and reveal improved properties. And also these dopants are introduced as substitutional defects on zinc sites. The fact that the radius of 4-coordinate $\mathrm{Ga}^{3+}(0.62 \AA)$ and that of $\operatorname{In}^{3+}(0.81 \AA)$ is closer to that of 4-coordinate $\mathrm{Zn}^{2+}(0.74 \AA)$, make it useful as a codopant for $\mathrm{ZnO}$ structures $[10,11]$. Thin film deposition of $\mathrm{ZnO}$ can be carried out by various techniques like sputtering [12], pulsed laser deposition [13], microwave assisted synthesis [14], sol-gel [15], ultrasonic spray pyrolysis [16], chemical bath deposition [17], etc. Among these, radio frequency (RF) sputtering is more suitable for high quality and large area deposition. 
In this paper, pristine $\mathrm{ZnO}$, indium-doped (IZO), galliumdoped (GZO) and indium and gallium co-doped ZnO (IGZO) thin films were deposited via RF sputtering techniques. All three of these materials were synthesized under the same conditions, which have allowed for an unprecedented and thorough comparison of the three doped materials. The effects of doping were investigated with respect to the crystal and grain growth of the films, and the resultant luminescence properties.

\section{Experimental}

\subsection{Film preparation}

Thin films of pristine $\mathrm{ZnO}, \mathrm{ZnO}$ with $\mathrm{In}\left(\mathrm{Zn}_{0.99} \mathrm{In}_{0.01} \mathrm{O}\right)$ (IZO) and $\mathrm{Ga}\left(\mathrm{Zn}_{0.99} \mathrm{Ga}_{0.01} \mathrm{O}\right)(\mathrm{GZO})$ as a single dopant and indium and gallium $\left(\mathrm{Zn}_{0.98} \mathrm{In}_{0.01} \mathrm{Ga}_{0.01} \mathrm{O}\right.$ ) (IGZO) as co-dopants were deposited on glass substrates using RF magnetron sputtering using powder targets. Sputter targets were prepared using a mixture of high purity $\mathrm{ZnO}$ powder and the desired amount of high purity $\mathrm{In}_{2} \mathrm{O}_{3}$ and $\mathrm{Ga}_{2} \mathrm{O}_{3}$ powders. The mixture was ground thoroughly, spread on a copper holder and pressed using a stainless steel puncher. Prior to the deposition of films the glass substrates were ultrasonically cleaned with trichloroethylene, acetone, methanol and deionized water for 10 min each. The cleaned substrates were dried in air before being introduced into the sputtering chamber. The chamber was evacuated to a base pressure of $3 \times 10^{-5} \mathrm{mbar}$, and the substrates were then heated to a growth temperature of $100^{\circ} \mathrm{C}$. A mixture of high purity Ar and $\mathrm{O}_{2}$ gas $(99.99 \%)$ was used as the plasma source, and the gas flow rate was controlled at 20 sccm each using a mass flow controller. A RF power of $75 \mathrm{~W}$ and a working pressure of $1.5 \times 10^{-2}$ mbar were used for film deposition. The film deposition was carried out for $120 \mathrm{~min}$ and prior to the deposition pre-sputtering was carried out for 10 min to remove any contamination from the substrate.

\subsection{Characterization of the film}

The crystallinity of the films was examined by X-ray diffraction (XRD) using a D8 ADVANCE diffractometer. The surface morphology of the films was investigated by field emission scanning electron microscopy (FESEM, MakeNova) and the attached energy dispersive X-ray spectroscopy (EDS) was used to examine the lateral variation of the elements. The photoluminescence (PL) spectra were recorded by using a fluorescence spectrometer (FLSP 920, Edinburgh Instruments). The chemical states of the films were identified by using an X-ray photoelectron spectrometer (XPS) using $\mathrm{Mg}-\mathrm{K} \alpha \mathrm{X}$-ray beams as the excitation source $(1253.6 \mathrm{eV})$ and a DESA-150 electron analyser (Staib Instruments, Germany). Optical spectra were recorded using a UV-Vis Jasco V-530 in the wavelength range of 300 to $900 \mathrm{~nm}$. Raman spectroscopy was performed using a Vyon Labram HR800 Raman spectrometer with the $632 \mathrm{~nm}$ excitation line of a solid state laser in the range of $100-800 \mathrm{~cm}^{-1}$. All the measurements were performed at room temperature.

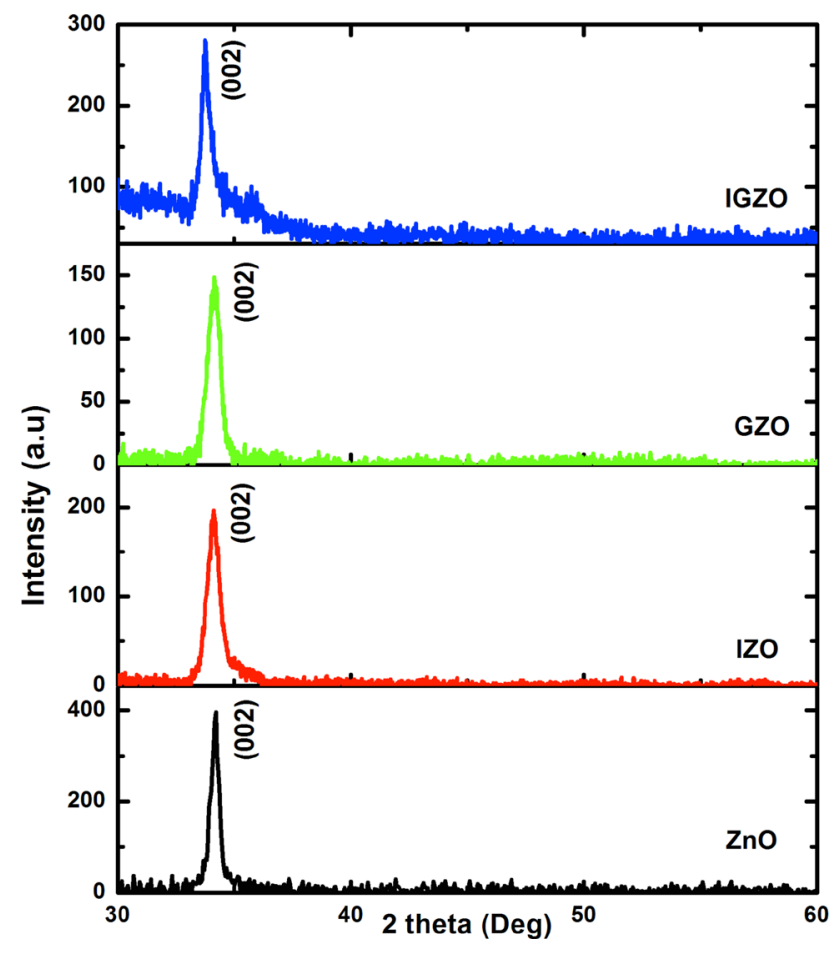

Figure 1. XRD patterns of pristine $\mathrm{ZnO}, \mathrm{IZO}, \mathrm{GZO}$ and IGZO nanocrystalline thin films.

\section{Results and discussion}

\subsection{Phase composition and microstructure}

The XRD patterns of pristine, single-doped (IZO and GZO) and co-doped (IGZO) $\mathrm{ZnO}$ thin films have only one strong (002) peak indicating that all films have a well-developed wurtzite structure and a good $c$-axis orientation perpendicular to the substrate (figure 1). No other peaks belonging to either gallium or indium phases are observed in the spectra, suggesting that both $\mathrm{Ga}$ and In are incorporated well into the $\mathrm{ZnO}$ lattice. It is observed that the doped-film shows a slight shift towards a lower angle compared to pristine $\mathrm{ZnO}$, which can be attributed to the difference in the ionic radius of $\mathrm{In}^{3+}$ $(0.81 \AA), \mathrm{Ga}^{3+}(0.62 \AA)$ and $\mathrm{Zn}^{2+}(0.74 \AA)$, respectively [18]. Also different shift directions of the (002) peak indicate different alternations of interplanar distances. The size of the crystallites (grain diameter) $D$ was calculated using Scherrer's formula [19]:

$$
D=k \lambda / \beta \cos \theta,
$$

where $k$ is the shape factor generally taken as $0.9, \lambda$ is the wavelength of $\mathrm{X}$-ray, $\beta$ is the full-width at half maximum (FWHM) value and $\theta$ is the XRD peak position. The values of lattice parameters ( $a$ and $c$ ) of the hexagonal wurtzite structure were calculated from the XRD peak using the equation [19]:

$$
a=\lambda / \sqrt{3} \sin \theta, \quad c=\lambda / \sin \theta,
$$


Table 1. Lattice parameters of single and co-doped $\mathrm{ZnO}$ thin films calculated along the (002) orientation.

\begin{tabular}{lccccccccc}
\hline Sample & $2 \theta$ & FWHM & $D(\AA)$ & $\begin{array}{c}\text { Dislocation } \\
\text { density }(\delta) \times 10^{15}\end{array}$ & $a(\AA)$ & $c(\AA)$ & $d(\AA)$ & $c / a$ & Strain $\left(10^{-3}\right)$ \\
\hline ZnO & 34.17 & 0.469 & 177 & 3.19 & $3.026 \pm 0.004$ & $5.241 \pm 0.008$ & $2.621 \pm 0.004$ & 1.732 & 1.96 \\
IZO & 34.12 & 0.729 & 114 & 7.70 & $3.031 \pm 0.002$ & $5.249 \pm 0.004$ & $2.625 \pm 0.002$ & 1.732 & 3.04 \\
GZO & 34.13 & 0.676 & 123 & 6.62 & $3.029 \pm 0.003$ & $5.248 \pm 0.005$ & $2.624 \pm 0.003$ & 1.732 & 2.82 \\
IGZO & 33.81 & 0.759 & 109 & 8.38 & $3.057 \pm 0.006$ & $5.295 \pm 0.007$ & $2.648 \pm 0.008$ & 1.732 & 3.17 \\
\hline
\end{tabular}

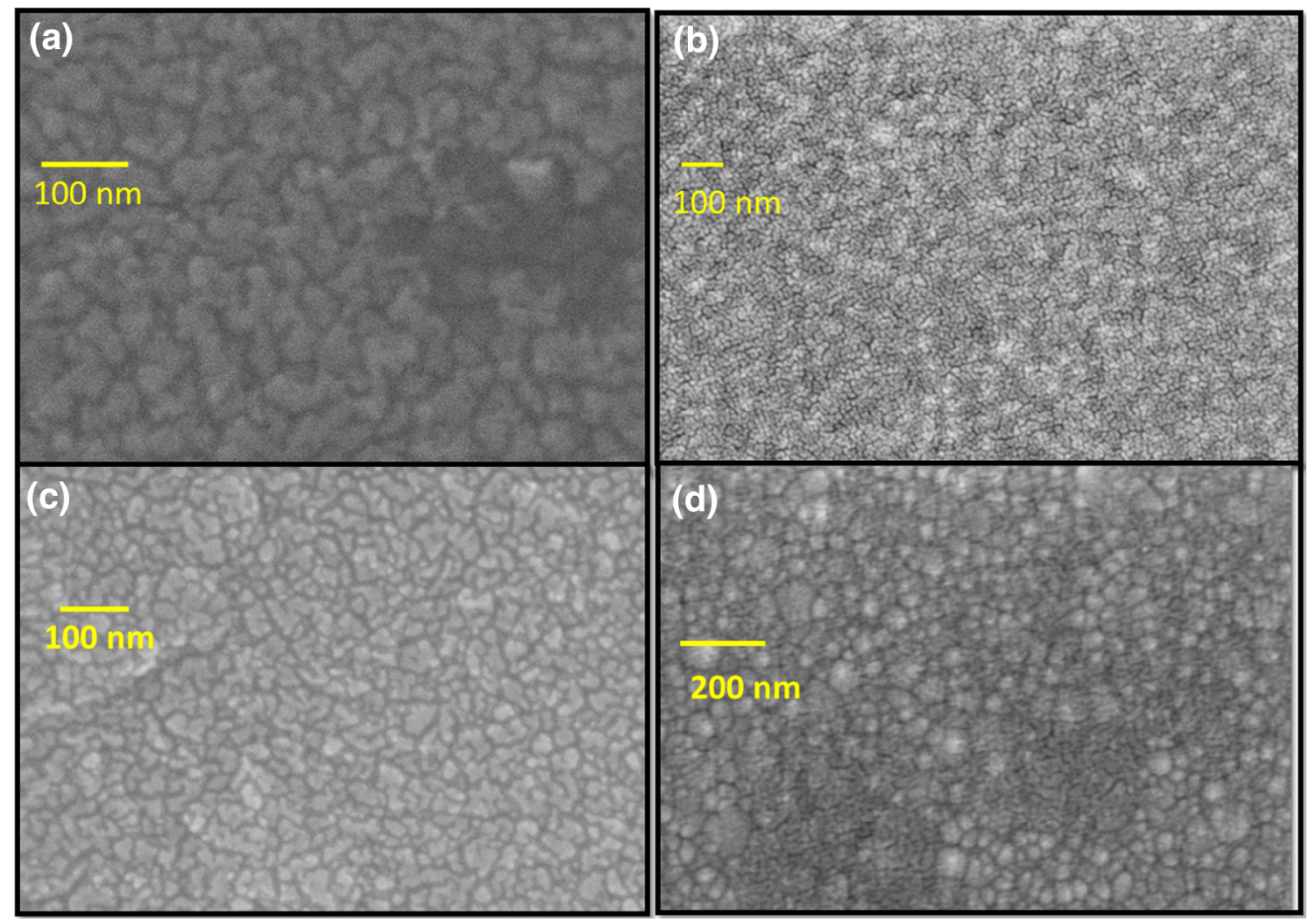

Figure 2. FESEM micrograph of: (a) $\mathrm{ZnO}$, (b) IZO, (c) GZO and (d) IGZO nanocrystalline thin films.

where values of $a$ and $c$ were estimated to be 3.026 and $5.248 \AA$ A respectively, with a $c / a$ ratio of 1.732 .

The average lattice strain $(\varepsilon)$ of the single and co-doped $\mathrm{ZnO}$ thin films can be obtained from the following expression [20]:

$$
\varepsilon=\beta \cos \theta / 4 \text {. }
$$

And the dislocation density $(\delta)$ is calculated from the relationship [20]:

$$
\delta=1 / D^{2}
$$

The grain size and lattice parameters, dislocation density and strain developed in the film are tabulated in table 1 .
It can be observed that the crystallite size of single and co-doped $\mathrm{ZnO}$ decreases from 177 to $109 \AA$. This could be attributed to the presence of a small strain and dislocations in the $\mathrm{ZnO}$ films due to the difference in the ionic radius of In $(0.81 \AA)$ and $\mathrm{Ga}(0.62 \AA)$ dopants. The data reveal that the presence of In and $\mathrm{Ga}$ ions in $\mathrm{ZnO}$ prevents the growth of crystal grains. The calculated crystal defect parameters like microstrain and dislocation density indicate an increasing trend with doping while the crystallite size decreases due to the replacement of $\mathrm{Zn}^{2+}(0.74 \AA)$ by $\mathrm{In}^{3+}$ $(0.81 \AA)$ and $\mathrm{Ga}^{3+}(0.62 \AA)$. In the case of IGZO, it is possible that the simultaneous incorporation of both dopants in the lattice structure is leading to a compensation effect, and the difference in the size of the two dopants helps in reducing some of the crystal strain, in turn allowing greater dopant incorporation in the $\mathrm{ZnO}$ structure. 
Figure 2 shows the FESEM images of pristine and doped-ZnO films. All the films have a dense, uniform, smooth and crack free microstructure. The morphology of $\mathrm{ZnO}$ and GZO has a granular structure while that of IZO shows a cornlike structure. The grain size of the IGZO thin films is smaller than that of $\mathrm{ZnO}$ which is attributed to the various relationships between the nucleation, growth and activation energy of the thin films $[18,20]$. The ionic radius inequality between $\mathrm{Ga}^{3+}(0.62 \AA), \mathrm{In}^{3+}(0.81 \AA)$ and $\mathrm{Zn}^{2+}(0.74 \AA)$ in the lattice site of $\mathrm{ZnO}$ has played a decisive role in the overall surface morphology modification of the films. Owing to the close ionic radius disparity between $\mathrm{Ga}^{3+}$ and $\mathrm{In}^{3+}$, the compression in the volume caused by gallium to the $\mathrm{ZnO}$ lattice is effectively compensated and counteracted by the tension in the lattice caused by indium such that there will be less strain imposed in the lattice and fewer dislocations will be formed [20]. As a result, IGZO films have a better surface morphology than other films.

\subsection{Elemental analysis}

The weight $\%$ of all elements of the thin films is studied with the help of EDS and is summarized in table 2. The

Table 2. Compositional ratio of $\mathrm{ZnO}$, IZO, GZO and IGZO thin films from EDS.

\begin{tabular}{lccccc}
\hline Sample & Zn (wt\%) & O (wt\%) & In (wt\%) & Ga (wt\%) & Total \% \\
\hline ZnO & 52.2 & 47.8 & - & - & 100 \\
IZO & 68.8 & 29.5 & 1.7 & - & 100 \\
GZO & 58.6 & 41 & - & 0.4 & 100 \\
IGZO & 81.4 & 18 & 0.4 & 0.2 & 100 \\
\hline
\end{tabular}

associated spectra obtained by EDS studies of all films are shown in figure $3 \mathrm{a}-\mathrm{d}$. EDS studies confirm the presence of doped elements such as In and $\mathrm{Ga}$ in $\mathrm{ZnO}$. The amount of the doped elements are found to be less than the actual amount of target composition.

In order to understand the oxidation states of the deposited thin films, XPS analyses are performed and shown in figures 4 and 5 . The spectra are corrected using the $\mathrm{C} 1 \mathrm{~s}$ line from adventitious aliphatic carbon $(285 \mathrm{eV})$. It is seen that the $\mathrm{O} 1 \mathrm{~s}$ and $\mathrm{Zn} 2 \mathrm{p}$ peaks of the doped- $\mathrm{ZnO}$ thin films (figure $4 \mathrm{a}$ and b) shift towards higher B.E.s compared to pristine $\mathrm{ZnO}$ thin films. This attributed to the formation of stronger bonds between the dopants (In and $\mathrm{Ga}$ ) and oxygen in doped-thin films compared to those between $\mathrm{Zn}$ and oxygen in the pristine $\mathrm{ZnO}$. The B.E. of the zinc $2 \mathrm{p}_{3 / 2}$ and $2 \mathrm{p}_{1 / 2}$ peaks (figure $4 \mathrm{~b}$ ) of all the films is consistently found to be $1020.2 \pm 0.2$ and $1043.2 \pm 0.2 \mathrm{eV}$ respectively which can be attributed to $\mathrm{Zn}^{2+}$ in $\mathrm{ZnO}$. The $\mathrm{O} 1 \mathrm{~s}$ spectra of all the films can be deconvoluted into three distinct sub peaks (I, II and III) using Gaussian fitting (figure 4a). Among them, the low energy (region I) peak $(529.4 \pm 0.2 \mathrm{eV})$ originates from $\mathrm{O}^{2-}$ ions forming bonds with the metal cations $\left(\mathrm{Zn}^{2+}, \mathrm{In}^{3+}\right.$ and $\left.\mathrm{Ga}^{3+}\right)$ in the lattice. The medium energy region II (530.4 $\pm 0.5 \mathrm{eV})$ components are associated with $\mathrm{O}^{2-}$ ions near the oxygen deficient regions within the $\mathrm{ZnO}$ lattice and region III $(532.01 \pm 0.5 \mathrm{eV})$ is due to the presence of loosely bound oxygen on the film surface belonging to a specific ratio such as $\mathrm{CO}_{3}$, adsorbed $\mathrm{H}_{2} \mathrm{O}$ or adsorbed oxygen [14,21-23]. The In $3 \mathrm{~d}$ and Ga $3 \mathrm{~d}$ peaks are clearly seen from the XPS survey spectrum of IZO, GZO and IGZO thin films, indicating that In and Ga have indeed blended into the $\mathrm{ZnO}$ lattice. The gallium $3 \mathrm{~d}_{5 / 2}$ peaks of $\mathrm{GZO}$ (figure 5b) and IGZO films (figure 5c) have B.E. of 20.33 and $20.31 \mathrm{eV}$, respectively, which is due to $\mathrm{Ga}^{3+}$. Similarly, the indium $3 \mathrm{~d}_{5 / 2}$ and $3 \mathrm{~d}_{3 / 2}$ peaks of IZO (figure $5 \mathrm{a}$ ) and IGZO
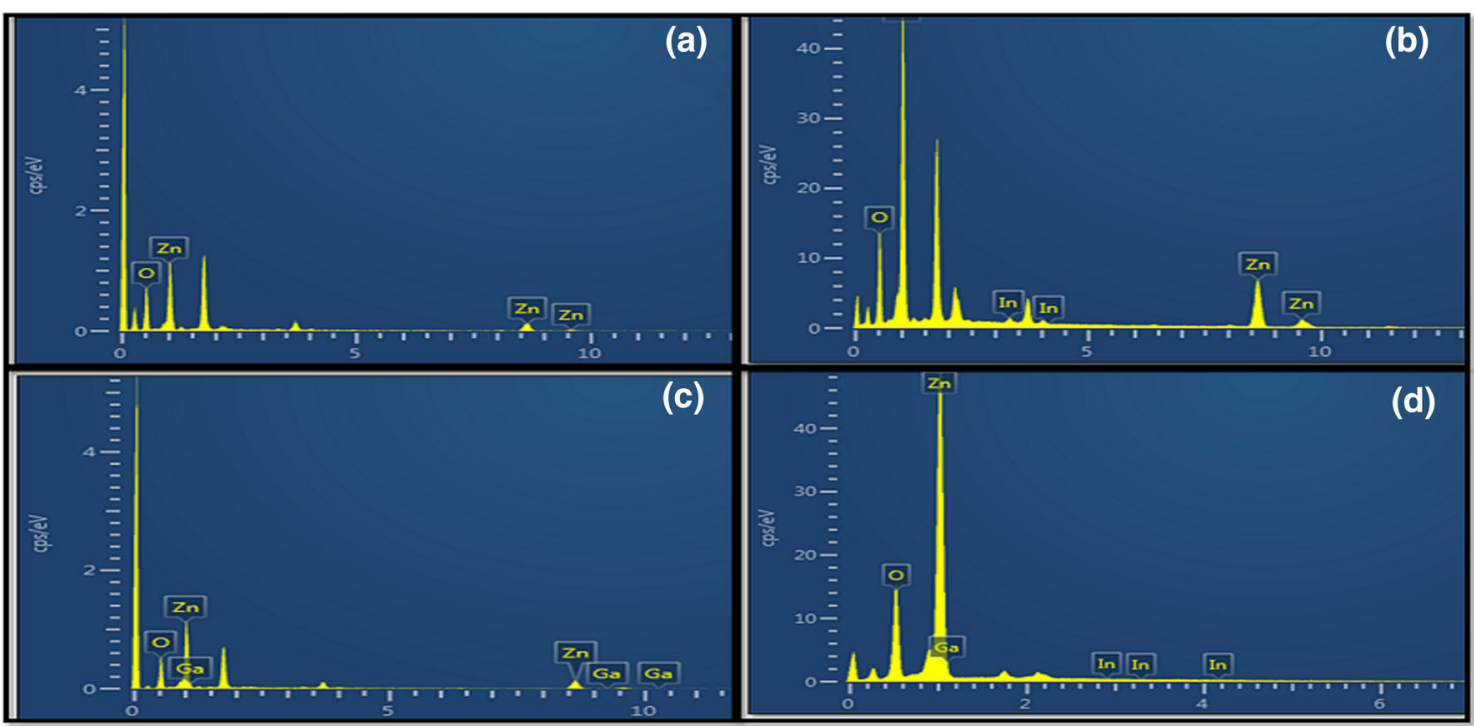

Figure 3. EDS spectra of: (a) $\mathrm{ZnO}$, (b) IZO, (c) GZO and (d) IGZO nanocrystalline thin films. 

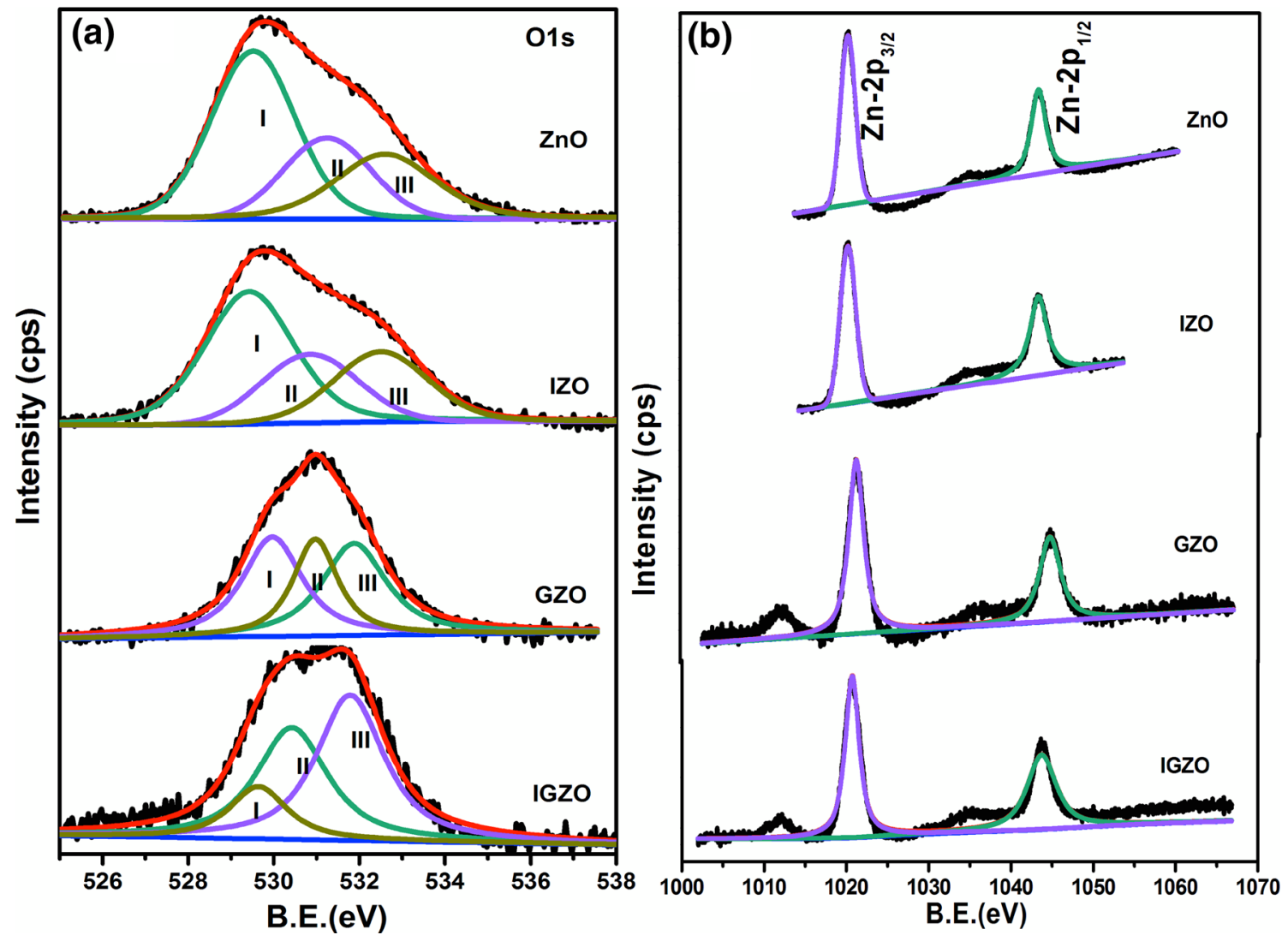

Figure 4. XPS analysis: (a) deconvoluted $\mathrm{O} 1 \mathrm{~s}$ spectra and (b) $\mathrm{Zn} 2 \mathrm{p}$ spectra of $\mathrm{ZnO}$, IZO, GZO and IGZO thin films.

(figure 5d) have B.E. of 444.4 and $451.5 \mathrm{eV}$ and 444.9 and $452.29 \mathrm{eV}$ respectively, which is due to $\mathrm{In}^{3+}$ [14].

\subsection{Optical properties}

The optical absorbance spectra of ZnO, IZO, GZO and IGZO thin films are shown in figure 6a. The well distinguishable peak in the UV range of the absorbance spectrum may be due to the exciton band of $\mathrm{ZnO}$. The optical transmittance spectra of the deposited films are shown in figure 6b. All deposited films exhibit an average transmittance of $>90 \%$ in the visible region and a sharp cut off between 360 and $375 \mathrm{~nm}$. The transmittance at $540 \mathrm{~nm}$ of $\mathrm{ZnO}, \mathrm{IZO}, \mathrm{GZO}$ and IGZO films is found to be $80,95,89$ and $83 \%$, respectively. Among all the films, IZO shows the highest transmittance. It is evident that doping affects the transmittance of the films only marginally. This may be due to the formation of a grainy surface leading to the large scattering loss. The absorption edge of the IGZO film is shifted towards a lower wavelength. This shift is responsible for the improved transmittance in the lower wavelength region as compared to that of the pristine $\mathrm{ZnO}$ thin films. An interference fringe can be observed in each spectrum, indicating that the deposited films have a very smooth and homogeneous surface.
The optical band gap $\left(E_{\mathrm{g}}\right)$ of $\mathrm{ZnO}$, IZO, GZO and IGZO thin films is computed using linear extrapolation of $(\alpha h v)^{2}$ $v s . h v$ plot. The absorption coefficient $\alpha$ has been calculated using Lambert's law for directly allowed transition [24]

$$
(\alpha h v)^{2} \propto\left(h v-E_{\mathrm{g}}\right)^{1 / 2},
$$

where $h v$ is the incident photon energy and $E_{\mathrm{g}}$ is the optical band gap. The optical band gap of the films is found to be dependent on the doping. The band gap of IZO (3.20 \pm $0.02 \mathrm{eV}$ ) and IGZO $(3.16 \pm 0.04 \mathrm{eV})$ films decreases (red shift) whereas for GZO films $(3.31 \pm 0.03 \mathrm{eV})$ it increases (blue shift) as compared with that of $\mathrm{ZnO}(3.24 \pm 0.01 \mathrm{eV})$. This variation could be due to the increase in the carrier concentration, known as the Burstein-Moss effect [25].

\subsection{PL spectra}

Figure 7a-d illustrates the room temperature PL spectra of pristine and doped-ZnO thin films for an excitation wavelength of $325 \mathrm{~nm}$. The spectral peak of all the samples is broad in the range of $350-600 \mathrm{~nm}$. This broad visible band is associated with deep level emissions due to intrinsic point defects and defect complexes in the film structure. The fitted PL spectra of IZO (figure 7b) and GZO (figure 7c) consist of 

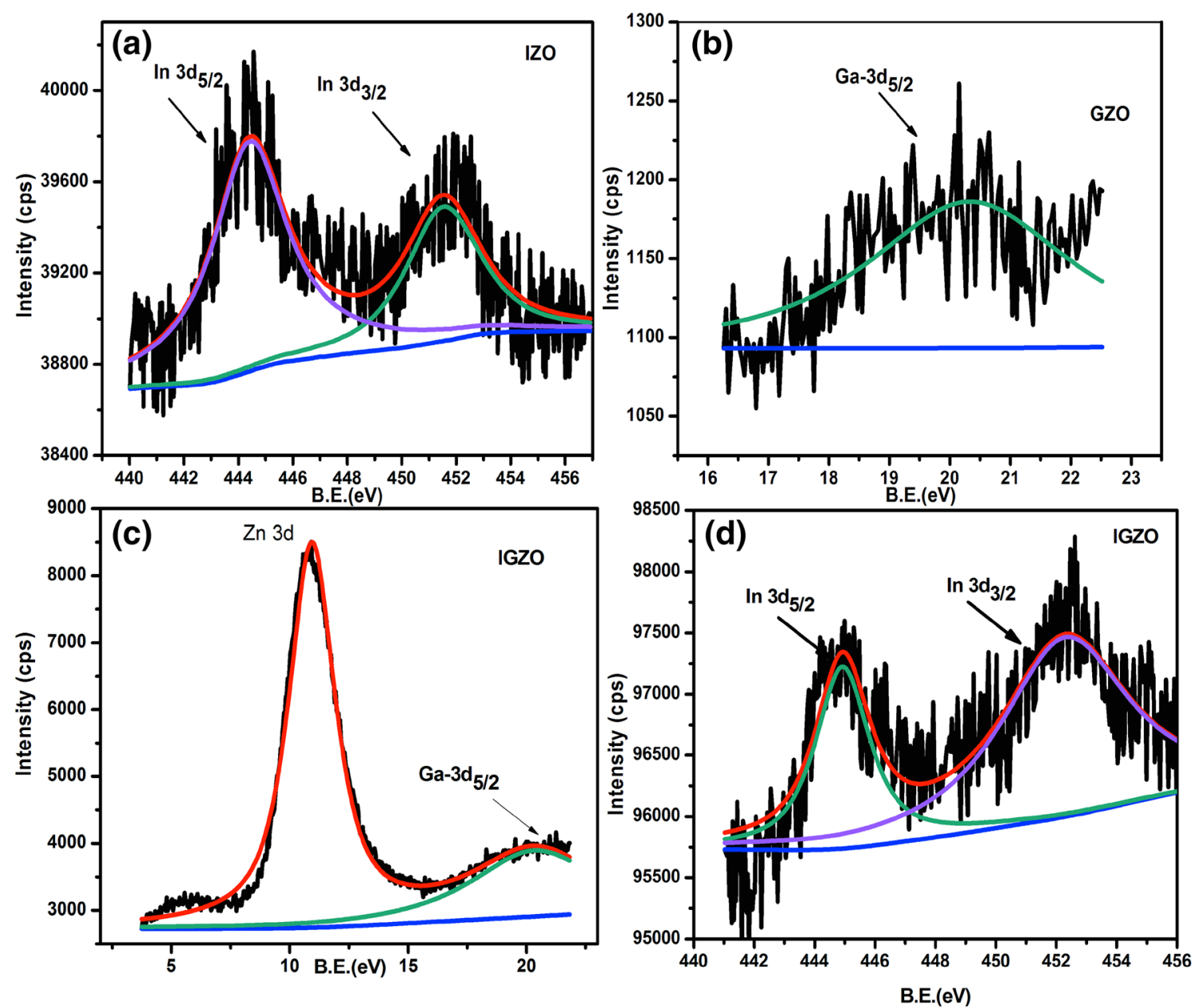

Figure 5. XPS spectra of: (a) In $3 d$ in IZO, (b) Ga $3 d_{5 / 2}$ in GZO and (c and d) In $3 d$ and Ga $3 d_{5 / 2}$ in IGZO thin films.
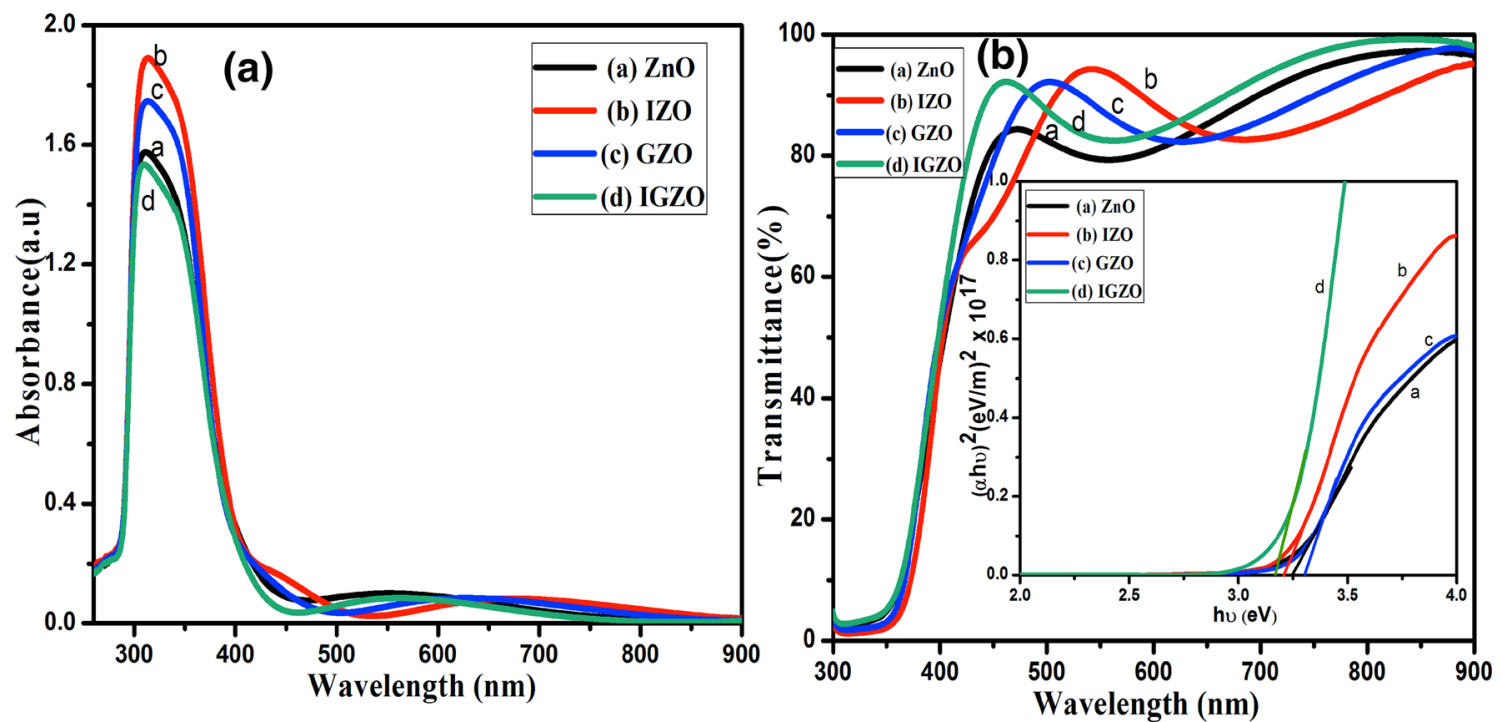

Figure 6. UV-visible (a) absorbance spectra and (b) transmittance spectra in the wavelength range of 300-900 nm and the inset shows the plot of $(\alpha h v)^{2} v s$. $h v$ of the pristine, single and co-doped $\mathrm{ZnO}$ thin films. 

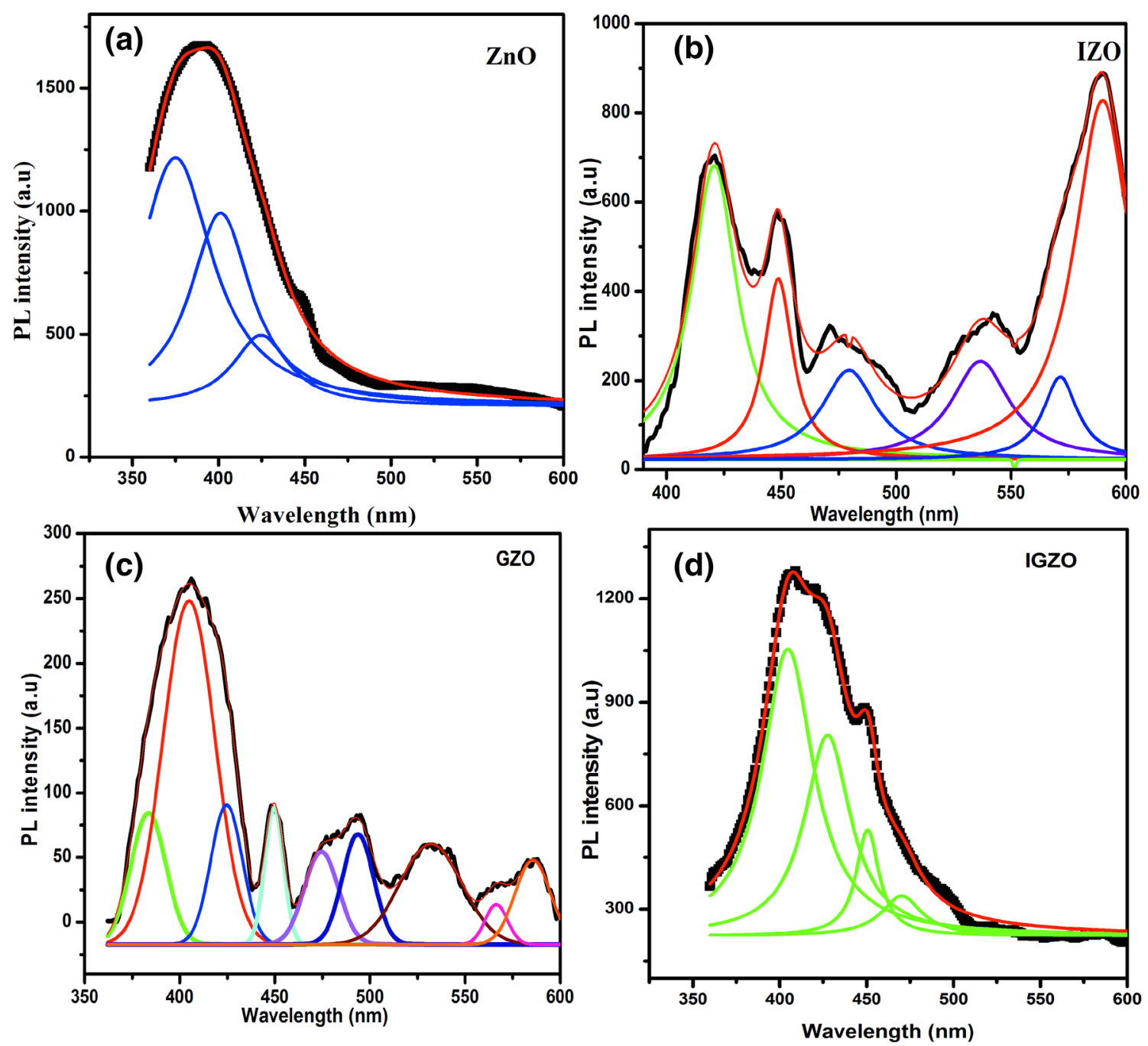

Figure 7. Deconvoluted room temperature PL spectra of (a) $\mathrm{ZnO}$, (b) IZO, (c) GZO and (d) IGZO thin films (excitation at $325 \mathrm{~nm})$.

several peaks. The orange-red emission is attributed to the deep interstitial oxygen (Oi) states inside the IZO structures. The yellowish-green emission is attributed to the transition from the bottom of the conduction of IZO and GZO structure to the Oi states, and the green emission is recognized as the $V_{\mathrm{O}}$ defects in both the structures [21]. The intensity of the orange-red emission is enhanced after indium doping, implying that the density of Oi states is increased in the IZO film. All the films exhibit the UV peak, which is associated with the free exciton-exciton collision process and is attributed to the near band edge emission (NBE) [26]. The pristine $\mathrm{ZnO}$ thin film exhibits an emission band ranging from 360 to $450 \mathrm{~nm}$ with a peak centred at $375 \mathrm{~nm}$ (figure 7a). For IGZO, (figure 7d) the emission band (ranging from 375 to $520 \mathrm{~nm}$ ) is slightly shifted towards the visible region due to the incorporation of dopants with the peak centred at $420 \mathrm{~nm}$. The PL spectra of IGZO thin films contain multiple peaks between 420 and $480 \mathrm{~nm}$. These violet-blue and blue emissions are attributed to zinc interstitials $\left(\mathrm{Zn}_{\mathrm{i}}\right)$ and zinc vacancy $\left(\mathrm{V}_{\mathrm{zn}}\right)$, respectively, explaining the change in emission during doping $[27,28]$. From the PL characteristics, it is clearly evident that the co-doped IGZO material is a potential candidate for use in down converting LEDs.

\subsection{Raman analysis}

In order to crease information regarding the crystallinity and phase of the single and co-doped films, Raman measurement of the samples (on Si substrates) is taken. Figure 8a shows the Raman spectra of single and co-doped $\mathrm{ZnO}$ thin films. The Raman lines around $437 \mathrm{~cm}^{-1}$ are observed for all the thin films, which is assigned to E2 (high) mode. This mode is assigned to the vibration of the oxygen sublattice and is the characteristics peak of the hexagonal wurtzite phase of $\mathrm{ZnO}$ films. In doped films, the E2 (high) mode is slightly blueshifted from 437 to $436.4 \mathrm{~cm}^{-1}$, caused by the tensile strain in the films which is in good agreement with the XRD result. A weak peak at $589 \mathrm{~cm}^{-1}$ (figure $8 \mathrm{~b}$ ) corresponds to A1 (LO) 

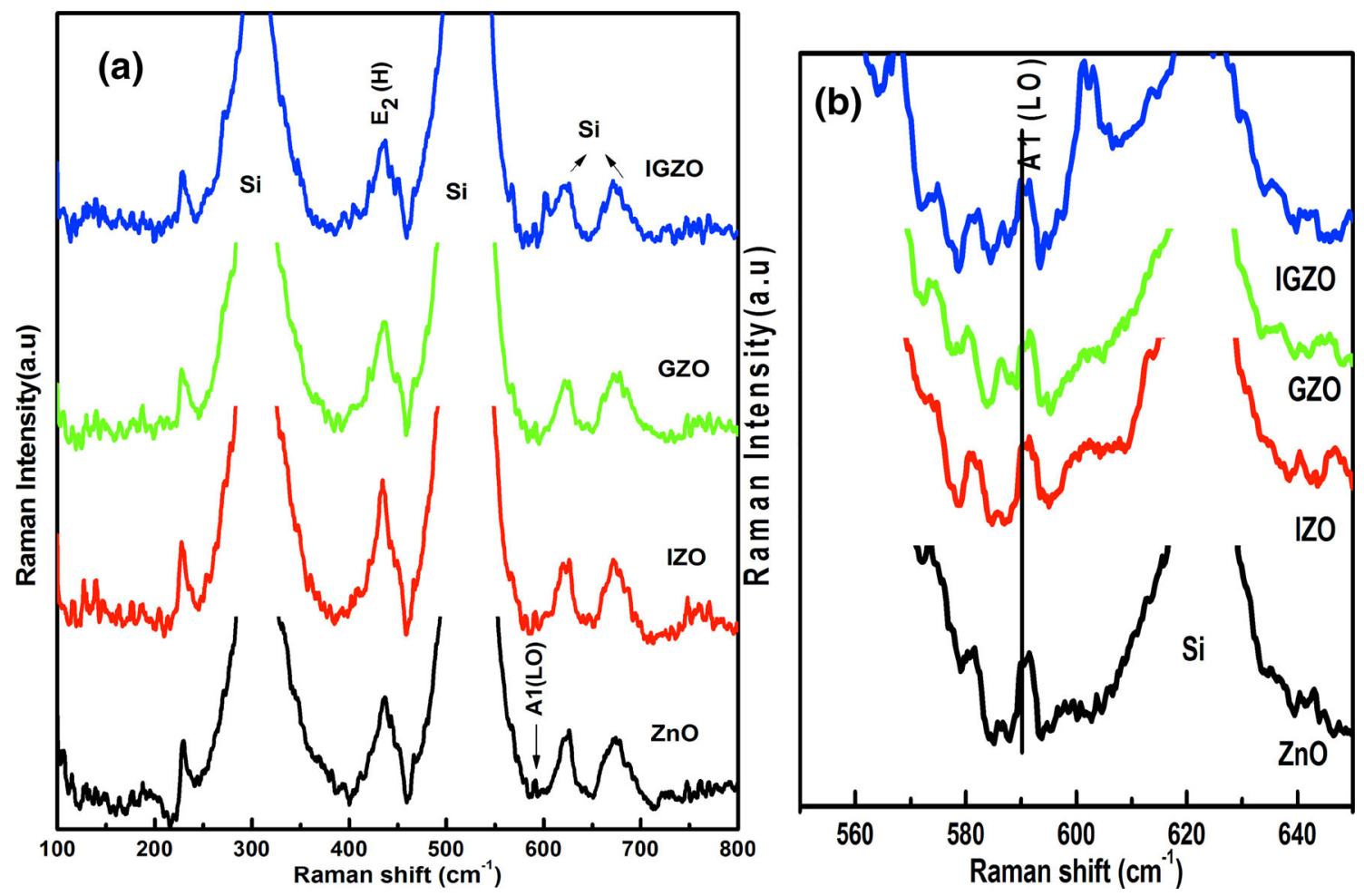

Figure 8. (a) Raman spectra and (b) enlarged view of single and co-doped $\mathrm{ZnO}$ thin films.

mode and may be caused by the defects such as O-vacancy and $\mathrm{Zn}$ interstitial and their complexes, which is consistent with the PL and XPS results.

\section{Conclusion}

Pristine, single (In, Ga) and co-doped $\mathrm{ZnO}$ thin films were deposited on glass substrates by RF magnetron sputtering. All the thin films exhibited a typical wurtzite structure with a strong (002) preferred orientation. XPS analysis confirmed the presence of the oxidized dopants in each film. The tensile strain in the films that caused the Raman line shift was in good agreement with that obtained from the XRD result. The peak attributed to the oxygen vacancy defect in Raman spectra is consistent with that observed from PL spectra and XPS. Among the doped films, the IGZO film showed better morphology, crystallinity and PL emission. All the films had very high-optical transmittance of the order of $95 \%$ in the visible range. The excellent optical properties reveal a great promise for the proposed thin films with applications in various optoelectronic devices.

\section{Acknowledgements}

The first and fourth authors would like to thank the thin film devices group, Technical Physics Division at BARC, Mumbai, for their help during the work.

\section{References}

[1] Jayaraman V K, Alvarez A M, de la luz M and Amador O 2017 Physica E 86164

[2] Wang F-H and Chang C-L 2016 Appl. Surf. Sci. 370 83

[3] Snigurenko D, Guziewicz E, Krajewski T A, Jakiela R, Syryanyy Y, Kopalko K et al 2016 Mater. Res. Express 3 125907

[4] Li X, Yang Y, Wu X, Zhang F C and Yang H 2018 Ferroelectr. $\mathbf{5 3 0} 11$

[5] Shahid M U, Deen K M, Ahmad A, Akram M A, Aslam M and Akhtar W 2016 Appl. Nano Sci. 6235

[6] Castro M V and Tavares C J 2015 Thin Solid Films 586 13

[7] You H-C 2013 Int. J. Electrochem. Sci. 89773

[8] Al-Asedu H J, Bidis N, Al Khafaji S A and Bakhtiar H 2018 Mater. Sci. Semicond. Proc. 7750

[9] Benhaliliba M, Benouis C E, Aida M S, Yakuphanoglu F and Sanchez Juarez A $2010 \mathrm{~J}$. Sol-Gel Sci. Technol. 55 335

[10] Chang S-C 2014 Nanoscale Res. Lett. 9562

[11] Chang S-C 2014 Int. J. Photoenergy 2014916189

[12] Gaspara D, Pereiraa L, Gehrke K, Galler B, Fortunato E and Martins R 2017 Sol. Energy Mater. Sol. Cells 163 255

[13] Singh K, Dhar R and Mohan D 2016 J. Integr. Sci. Technol. 4 33

[14] Jayathilake D S Y, Nirmal Peiris T A, Sagu J S, Potter D B, Wijayantha K G U, Carmalt C J et al 2017 ACS Sustain. Chem. Eng. 54820 
[15] Wahab H A, Salama A A, El-Saeid A A, Nur O, Willande M and Battisha I K 2013 Results Phys. 346

[16] Jayaraman V K, Álvarez A M, Kuwabara Y M, Koudriavstev Y, de la luz M and Amador O 2016 Mater. Sci. Semicond. Proc. 4732

[17] Bidier S A, Hashim M R, Al-Diabat A M and Bououdina M 2017 Physica E 88169

[18] Le H Q and Chua S J 2011 J. Phys. D: Appl. Phys. 44125104

[19] Gadallah A S and El Nahass M M 2013 Adv. Cond. Mat. Phys. 2013 Article ID 234546

[20] Rouchdi M, Salmani E, Fares B, Hassanain N and Mzerd A 2017 Results Phys. 7620

[21] Chirakkara S and Krupanidhi S B 2011 Phys. Status Solidi RRL 634
[22] Wang F-H, Chao J-C, Liu H-W and Kang T-K 2015 J. Nanomater 2015936482

[23] Duan J, Xiong Q, Hu J and Wang H 2015 J. Power Energy Eng. 311

[24] Zhu Y, Mendelsberg R J, Zhu J, Han J and Anders A 2013 Appl. Surf. Sci. 265738

[25] Tohsophon T, Wattanasupinyo N, Silskulsuk B and Sirikulrat N 2013 Thin Solid Films 520726

[26] Abbasi M A, Ibupoto Z H, Hussain M, Nur O and Willander M 2013 Nanoscale Res. Lett. 8320

[27] Allabergenov B, Chung S-H, Jeong S M, Kim S and Choi B 2013 Opt. Mater. Express 31733

[28] Alvi N H, Ul Hasan K, Nur O and Willander M 2011 Nanoscale Res. Lett. 6130 